\title{
Evaluation of an enzyme-linked immunoassay and confirmatory test for the detection of Chlamydia trachomatis in male urine samples
}

\author{
R S Matthews, P G Pandit, S D Bonigal, R Wise, K W Radcliffe
}

\begin{abstract}
First pass urine (FPU) samples were compared with urethral swab culture from 304 males attending a genitourinary medicine clinic using an enzyme immuno assay (EIA). All of the EIA positive samples were retested by incorporating a novel blocking reagent into the EIA protocol; 101 were positive by culture of which 83 were also positive by FPU EIA, an additional four were detected in FPU only and not by culture; 86 of these 87 were also confirmed positive by the blocking reagent. Discrepant results were evaluated by Syva MicroTrak. The sensitivity and specificity of FPU EIA as compared with urethral swab culture was $82 \cdot 2 \%(83 / 101)$ and $98 \%$ (199/203) respectively with positive and negative predictive values of $95.4 \%(83 / 87)$ and $91 \cdot 7 \%$ (199/217). Male urethral swab culture is more sensitive than FPU EIA; however, when culture is not available then FPU offers a reliable non-invasive alternative to swab EIA which may be of enormous benefit in community screening of asymptomatic as well as symptomatic patients.
\end{abstract}

(Genitourin Med 1993;69:47-50)

\section{Introduction}

The reference standard for the detection of Chlamydia trachomatis from urogenital samples is by tissue culture. Although this is by no means $100 \%$ sensitive it is still the "benchmark" by which other methods are assessed. However, when utilising first pass urine (FPU) samples as an antigen source, tissue culture is of little value and enzyme immunoassay (EIA) and direct immunofluorescence (DIF) are the methods of choice. ${ }^{1-3}$ A difficulty arises when investigating FPU as a suitable alternative to urethral swabs as to which samples and methods are employed to compare results. Since our original study where urethral swab culture was compared with FPU amplified EIA, ${ }^{4}$ the relative merits of these techniques have been evaluated by many investigators using various 'benchmarks'. ${ }^{\text {-7 }}$ In this study we have compared the urethral swab culture status with FPU conventional EIA (AntigEnz Chlamydia, Shield Diagnostics Ltd., Dundee, U.K.) and confirmed the EIA positives with a novel blocking reagent (AntigEnz Chlamydia Confirmatory Test, Shield Diagnostics Ltd.) and DIF. In a multicentre study using urogenital swabs, the AntigEnz Chlamydia EIA test has been shown to have a sensitivity of $87 \%$ in men and $86 \%$ in women, with specificities of $94 \%$ and $97 \%$ respectively when compared with swab culture. ${ }^{8}$

\section{Methods}

Specimen collection

Urethral swabs and FPU samples were collected from 304 males with symptoms of dysuria and/or urethral discharge, attending the Department of Genitourinary Medicine, General Hospital, Birmingham over a 10 week period. In the first group (A) of 151 patients the urethral swabs were collected prior to the urine samples and in the second group (B) of 153 patients, this procedure was reversed. The urethral swab was placed in $2 \mathrm{ml}$ of chlamydia culture transport medium and both the FPU and urethral swab were stored at $+4^{\circ} \mathrm{C}$ for no longer than 24 hours before transportation to the laboratory.

\section{Laboratory procedures}

The urethral swabs were vortex mixed and inoculated onto two coverslip cultures of McCoy cells (ICN Flow, High Wycombe, UK) treated with cycloheximide and incubated at $35^{\circ} \mathrm{C}$ for $48-72$ hours. After fixing one of the coverslip cultures in methanol for 10 minutes it was stained by a group specific chlamydia monoclonal antisera (Imagen Dako Ltd., High Wycombe, UK) and examined by epifluorescence microscopy for typical intracytoplasmic inclusions. The remaining culture was passaged into fresh McCoy cells and incubated for a further 48 hours.

Any urines that were cloudy in appearance were warmed at $37^{\circ} \mathrm{C}$ to dissolve cold precipitins prior to all being cultured for bacterial contaminants by inoculating onto blood agar using a standard $10 \mu \mathrm{l}$ loop and incubating aerobically at $37^{\circ} \mathrm{C}$ for 24 hours.

If excess urine had been passed, the volume was adjusted to $15 \mathrm{mls}$, and centrifuged at $2,500 \mathrm{~g}$ for 20 minutes, the supernatant discarded and deposit resuspended in $1 \mathrm{ml}$ of AntigEnz Chlamydia transport medium. After vortex mixing $200 \mu \mathrm{l}$ was removed from each sample and stored in microvials at $-70^{\circ} \mathrm{C}$. In the event of discrepant results these stored samples, together with remaining EIA and culture specimens, were examined for $C$ trachomatis elementary bodies (EBS) using species specific monoclonal antisera (Syva MicroTrak, Palo Alto USA). AntigEnz 
Table 1 Summary of results

\begin{tabular}{lcccll}
\hline Urethral culture & + & + & - & - \\
Urine EIA & + & - & + & - \\
& 83 & 18 & 4 & $199=304$ \\
\hline
\end{tabular}

Chlamydia was performed according to the manufacturer's protocol and all results were recorded photometrically.

\section{Results}

Bacterial cultures

Although a wide variation in results were recorded from the blood agar culture the majority were of "no growth" or "scanty skin flora". There were no specimens that were EIA positive and chlamydia culture negative that could not be confirmed as true positives, and none of those samples with bacterial growth caused any significant rise in the recorded optical density (OD).

\section{Chlamydia cultures}

There were seven swab samples that caused very slight toxicity in the first monolayers of both negative and positive cultures, but as none of these were thought toxic enough to warrant exclusion from the trial all of the collected samples were included.

In the first group of 151 patients, where the swab was taken first, the overall detection rate by both methods was $28 \cdot 4 \%(43 / 151)$ and by tissue culture alone $27 \cdot 1 \%(41 / 151)$. In the second group of 153 , where the urine was collected first the detection rates were $40.5 \%(62 / 153)$ and $39 \cdot 2 \%(60 / 153)$ by tissue culture. There were two cases where the chlamydia culture was only positive after subculture, both were in group $B$ and both were also positive by urine EIA. As shown by ourselves and other workers, ${ }^{2}{ }^{4}$ the order of collection made no significant difference to the urine EIA sensitivity compared with positive urethral cultures, being $82.9 \%(34 / 41)$ in the first group and $81.7 \%(49 / 60)$ in the second. A summary of the results is give in table 1 .

\section{Confirmation of positive results using blocking} antibody

In addition to the clinical evaluation of AntigEnz Chlamydia for urine testing a concurrent study utilising a novel blocking antibody was carried out on all of the positive EIAs. Briefly, a polyclonal rabbit antichlamydia serum and equivalent concentration of normal rabbit serum was added to two wells after the addition of the test sample. Those samples that had given an initial high OD reading ( $>2.0$ absorbance units) were diluted 1:11 with freshly boiled transport

Table 2 Four ELA positive/culture negative samples

\begin{tabular}{|c|c|c|c|c|c|c|}
\hline \multirow[b]{2}{*}{$\begin{array}{l}\text { Lab No. } \\
2810 \\
2963 \\
4041 \\
4568\end{array}$} & \multirow[b]{2}{*}{$\begin{array}{l}\text { Urine } \\
\text { EIA } \\
+ \\
+ \\
+ \\
+\end{array}$} & \multirow[b]{2}{*}{$\begin{array}{l}\text { EIA } \\
\text { Blocked } \\
\text { No } \\
\text { Yes } \\
\text { Yes } \\
\text { Yes }\end{array}$} & \multirow[b]{2}{*}{$\begin{array}{l}\text { Urethral } \\
\text { Culture } \\
\text { Neg } \\
\text { Neg } \\
\text { Neg } \\
\text { Neg }\end{array}$} & \multicolumn{3}{|l|}{ EBs Detected } \\
\hline & & & & $\begin{array}{l}\text { Stored aliquor } \\
\text { Yes } \\
\text { Yes } \\
\text { No } \\
\text { Yes }\end{array}$ & $\begin{array}{l}E L A \\
\text { N/a } \\
\text { N/a } \\
\text { Yes } \\
\text { N/a }\end{array}$ & $\begin{array}{l}\text { Swab } \\
\text { Yes } \\
\text { Yes } \\
\text { Yes } \\
\text { No }\end{array}$ \\
\hline
\end{tabular}

medium prior to retesting. A $50 \%$ or greater reduction in the OD reading in the blocked well, as compared to the control well, confirmed the presence of chlamydia antigen. Of the 89 specimens that were re-tested using the blocking reagent, 86 were confirmed as positive by blocking and in one there was insufficient sample remaining. In the other two specimens that did not block, one was culture positive but with no visible EBs in the stored aliquot and the other was culture negative but had EBs in the stored aliquot and remaining swab sample. All three had OD readings within the "grey zone" (the threshold plus 0.05 absorbance units), but with reproducibility being a signal of positivity, coupled with a finite amount of specimen remaining, they were classified as EIA/blocking test negative according to the manufacturer's protocol, even though they were proved positive by the other means.

\section{Discordant results}

Besides analysing the discordant results of EIA positive and culture negative samples it was considered that all the culture positive and EIA negative should also be examined. There were four of the former (table 2) and 18 of the latter (table 3 ).

Of the 22 samples that initially gave negative EIA results with positive cultures, four became EIA positive when repeated and all proved to be true positives by either or both the blocking test and stored aliquot.

Comparison of urine EIA with urethral swab culture

As there was no significant difference between the first and second groups of patients the overall detection rate by both methods was $34.5 \%$ (105/304) with $28.6 \%$ $(87 / 304)$ by urine EIA and $33 \cdot 2 \%(101 / 304)$ by urethral culture. The sensitivity of the urine EIA as compared with the urethral swab culture was $82.2 \%(83 / 101)$ with a specificity of $98 \%(199 / 203)$. The positive and negative predictive values were $95.4 \%$ $(83 / 87)$ and $91 \cdot 7 \%(199 / 217)$ respectively with overall agreement of $92.8 \%(282 / 304)$ (see table 4). After resolution of the discrepant results the sensitivity of the urine EIA rose slightly to $82 \cdot 8 \%(87 / 105)$. These results compare favourably with other EIA test evaluations on male FPU samples. ${ }^{4567}$

\section{Discussion}

In this study the sensitivity of tissue culture was $96 \cdot 2 \%(101 / 105)$ as compared with the

Table 3 Eighteen EIA negative/culture positive samples

\begin{tabular}{cllll}
\hline Samples & $\begin{array}{l}\text { Urethral } \\
\text { Culture }\end{array}$ & $\begin{array}{l}\text { Urine } \\
\text { EIA }\end{array}$ & EBs Detected \\
\cline { 4 - 5 } & Stored aliquot & Swab \\
\hline $8^{\star}$ & + & - & - & + \\
10 & + & - & + & + \\
\hline
\end{tabular}

*In two specimens the repeat EIA OD readings fell in the "grey zone" (the threshold plus 0.05 absorbance units) but as there was insufficient remaining sample to block in one and the other was negative on blocking they were classified as negative. 
Table 4 Urethral swab culture v urine EIA

\begin{tabular}{rlc}
\hline & \multicolumn{3}{l}{ Urethral swab culture } \\
\cline { 2 - 3 } & Positive & Negative \\
Urine EIA Positive & 83 & 4 \\
Negative & 18 & 199 \\
\hline
\end{tabular}

overall detection rate by both methods. If this new figure is used for comparison of the EIA sensitivity then the adjusted results are $79.8 \%(82.9 \%)$ in the first group and $78.6 \%$ $(81.7 \%)$ in the second (original results in brackets). This lower sensitivity represents a more accurate figure and should be considered when evaluating differing methods of detection. EIA is probably only $85-95 \%$ as sensitive as culture which, in turn is thought to detect only $80-90 \%$ of actual infections. ${ }^{9}$

Immunofluorescence on the other hand, is a very sensitive technique, whether used in a direct manner or as a confirmatory test. Thomas et al ${ }^{10}$ has recently shown that DIF on $1 \mathrm{ml}$ of FPU deposit was as sensitive as EIA and may be the most sensitive method for utilising FPU samples, a conclusion we also came to when investigating sterile pyuria, but for differing reasons. " However, the factors governing the choice of method for the detection of $C$ trachomatis is not only sensitivity but also cost and level of technical competence required to satisfy quality assurance. DIF may be the most sensitive method for FPU but it also requires the highest degree of skill, is time consuming and is often a task undertaken by senior staff with increased revenue consequences.

In this present study we also evaluated a simple confirmatory kit with two prediluted, blocking and control reagents that were added to the usual assay scheme when confirming previously detected positives.

All of the initial 89 EIA positives detected in FPU were confirmed either by culture or DIF and 86 were confirmed by the blocking test. It was noted that in those samples that gave an original high $O D$ reading and were diluted prior to retesting the strength of the new OD signal was not always reduced in proportion to the dilution and in a few cases actually increased. Although this phenomenon has been seen in other centres (personal communication, EO Caul) and is related to the initial volume of urine/antigen chosen, it had no detrimental effect on the outcome of the blocking tests.

The confirmation of true positives by an EIA blocking mechanism is to be encouraged in all laboratories and especially in those that cannot confirm by DIF on the remaining sample. This is particularly important in low prevalence populations where the positive predictive value can be very low. For this reason it would be useful to evaluate AntigEnz Chlamydia blocking reagent in a low prevalence group to see if these results can be maintained.

In our 1987 study using an amplified EIA system (IDEIA Dako Diagnostics Ltd) ${ }^{4}$ we concluded that urethral swab culture was superior to FPU EIA and this present study utilising conventional EIA technology, confirms this view. However, since then, the use of tissue culture in the United Kingdom for the detection of $C$ trachomatis has declined. The results of questionnaires by the National External Quality Assessment Scheme ${ }^{1213}$ show that the number of responding laboratories offering culture has fallen from 36 in 1988 to 24 in 1990. Moreover, only two of the 24 offered culture alone and the other 22 also performed EIA or DIF. In the same period of time, the number using EIA has risen from 105 to 135 and DIF from 141 to 154 .

The accepted use of EIA when screening large numbers of samples as a suitable, albeit less sensitive method than tissue culture, is a reflection of kit manufacturers' continued refinement and quality assurance of the product. Given this alternative it is not surprising that many laboratories have forsaken culture in favour of EIA.

It therefore follows that if chlamydia culture is not generally available for swab samples from male urethra then FPU should be considered for the advantages, not only for the patient, but also for the clinician and laboratory. From the patients' point of view there is no trauma or pain involved in collecting the specimen, which may then be supervised by nursing staff after the initial medical consultation. This relieves the clinician of swab collection duties and could create a greater throughput of patients. For the laboratory, FPU is a more uniform specimen that can be divided, examined by more than one method and removes the inherent "sampling error" often associated with inadequate swabbing. This is an important point when diagnosing genital chlamydial infection from males in the community; especially general practice, where there are often inappropriate swabs or transport medium available. Furthermore, utilising a non-invasive means of diagnosing $C$ trachomatis infection would be of enormous benefit in massscreening of asymptomatic men to provide hitherto unavailable epidemiological information as well as aiding the diagnosis of asymptomatic cases and thus the control of genital chlamydial infection.

The Chlamydia AntigEnz test gave good positive and negative predictive values ( $95.4 \%$ and $91.7 \%$ respectively) on a highly selected group of males attending a GUM clinic; however, caution should be taken when extrapolating these findings to a lower prevalence population. Taking all these factors into consideration and if EIA is the only method available for the detection of $C$ trachomatis from male urogenital specimens then there is little to be gained from taking urethral swabs when FPU samples will give the same, or slightly reduced, sensitivity.

1 Smith TF, Weed LA. Comparison of urethral swabs, urine and urinary sediment for the isolation of chlamydia. $\mathcal{f}$ Clin Microbiol 1975;2:134-5.

2 Hay PE, Thomas BJ, Gilchrist C, Palmer HM, Gilroy CB, Taylor-Robinson $D$. The value of urine samples tion of Chlamydia trachomatis. Genitourin Med 1991; 
67:124-8

3 Caul EO, Paul ID, Milne JD, Crowley T. Non-invasive sampling methods for detecting Chlamydia trachomatis. Lancet 1988;ii: $1246-7$

4 Jawad AJ, Manuel G, Matthews RS, Wise R, Clay JC. Evaluation of a genus-specific monoclonal antibody in an amplified enzyme-linked immunoassay in the detection of chlamydia in urine samples from men. Sex Trans Diseases 1990;17:87-9.

5 Chernesky M, Castriciano S, Sellors J, et al. Detection of Chlamydia trachomatis antigens in urine as an alternative to swabs and cultures. $\Im$ Inf Dis 1990;161:124-6.

6 Paul ID, Caul EO. Evaluation of three Chlamydia trachomatis immunoassays with an unbiased noninvasive chomatis immunoassays with an unbiased nonin
clinical sample. $\mathcal{F}$ Clin Microbiol 1990;28;220-2.

7 Genc M, Stary A, Bergman S, Märdh P-A. Detection of Chlamydia trachomatis in first-void urine collected from men and women attending a venereal clinic. APMIS 1991;99:455-9.
8 Clark A, Stamm WE, Gaydos C, Welsh L, Quinn TC, Schachter J, Moncada J. Multicentre evaluation of the AntigEnz Chlamydia EIA for diagnosis of $C$ trachomatis infection. $\mathcal{f}$ Clin Microbiol (in press)

9 Caul EO. Current topics in clinical virology. Public Health Laboratory Service 1991:205-3.

10 Thomas BJ, Gilchrist C, Hay PE, Taylor-Robinson D. Simplification of procedures used to test urine samples for Chlamydia trachomatis. F Clin Pathol 1991;44: 374-5.

11 Matthews RS, Bonigal SD, Wise R. Sterile pyuria and Chlamydia trachomatis. Lancet 1990;8711:385.

12 Malic A, Banker N. Summary of results from a questionnaire on methods used for the laboratory diagnosis of naire on methods used for the laboratory diagnosis of Quality Assessment Scheme. Feb 151988.

13 Summary of Results of the UK Laboratories Distribution Number 609 . Chlamydia trachomatis detection. National External Quality Assessment Scheme 609 Nov 20, 1990. 